Special Issue of the 8th International Advances in Applied Physics and Materials Science Congress (APMAS 2018)

\title{
Geometry and Inertia of the Human Body
}

\author{
W.S. ERDMANN* \\ Gdansk University of Physical Education and Sport, Department of Biomechanics and Sport Engineering, \\ Gdansk, Poland, EU
}

\begin{abstract}
The aim of this paper is to present geometric and inertial quantities of human body and its impact on movement. The external view of whole body and its parts is represented by the length of the line segments, e.g. during measuring of body height or by comparing of a step length to the length of lower extremities. Curvilinear measurements are used for determining the circumference of body parts. Body planar dimensions are taken into account when air resistance is calculated, while body volume is essential when buoyancy is characterized. Body angles are used for presentation of e.g. body posture. External dimensions can be obtained using anthropometric devices and image processing methods. Internal dimensions for living people can be obtained using such methods as computerized tomography or magnetic resonance imaging. Diagnostic images of the body are used for obtaining tissue data, especially area of surface of tissue image on body intersection. The multiplication by the height of a layer gives the volume of tissues. Inertial quantities are whole body, segment, and tissue mass (absolute and relative), moment of inertia of the whole body and its parts, location of a center of mass in given coordinate system. Values of quantities above are necessary for building the body model for transport analyses and biomedical engineering and also for ergonomic, sport, medicine and other purposes.
\end{abstract}

DOI: 10.12693/APhysPolA.135.961

PACS/topics: humans, body, geometry, inertia, applications

\section{Introduction}

It is difficult to perform analyses of human body from both external and internal reasons. Human body has irregular geometric shape. Therefore during the analyses substitution of human body with some simplified geometric and inertial models is often necessary. Body parts are often presented as regular geometric shapes. They are taken into account in some analyses as rigid solids with no movement of internal tissues and in other, more detailed analyses as flexible objects.

Body inertial data are: body mass, density, coordinates of center of mass, i.e. location of center of mass according to the acquired reference system, moment of inertia, which takes into account body mass and its distribution around axis of rotation. The irregular body shape does not help in calculation of above mentioned quantities.

The aim of the article is a description of geometry and inertia of the human body - their definition, methods and instrumentation for obtaining data, examples of applications.

\section{Geometry of the body}

\subsection{External body geometry}

Geometry of the body encompasses linear, planar and spatial dimensions. It is used in anthropometry for description of dimensions of human population, in engineering design for construction of furniture or vehicles,

*e-mail: wlodzimierz.erdmann@awf.gda.pl in sport analyses, e.g. when comparing of a step length to the length of lower extremities. Planar dimensions can be analyzed as a whole body surface (skin surface) or an area perpendicular to the direction of movement taken into account for calculation of drag, i.e. air or water resistance. The volume of the body is analyzed when buoyancy is investigated usually when the body is immersed into the water. Angular measurements in joints and also angles between trunk parts are performed during body posture analyses and during different kinds of movement.

Internal view of the body is used in physiology where muscle cross-section area of surface is used for assessment of muscle strength and volume of the heart gives information on body efficiency during an effort. Calculation of internal volume is also used for such other organs as lungs, stomach, bladder. In orthopedics internal data are used e.g. for obtaining dimensions of bony construction or for joint reconstruction. Internal view is also necessary for tissue data, especially for calculation of area of surface of tissues seen at the image of a body layer. These internal geometric dimensions are not of a regular shape therefore sometimes for presenting anatomical organs a finite element method (FEM) is used.

Linear dimensions of the whole body or of its parts are measured in different manners: (i) with the help of anthropometer, i.e. a narrow calibrated tube with horizontal arm that can move along a tube and perpendicular to it. The tube is based on a floor in the center and behind the feet, hold vertically and the end of an arm touches specific body landmark (Fig. 1a); (ii) using an image (a photography, a frame of a movie, or a video picture) where near the investigated body an object of known dimension is seen which helps to calculate a scale of a an image (Fig. 1b); (iii) introducing regular 


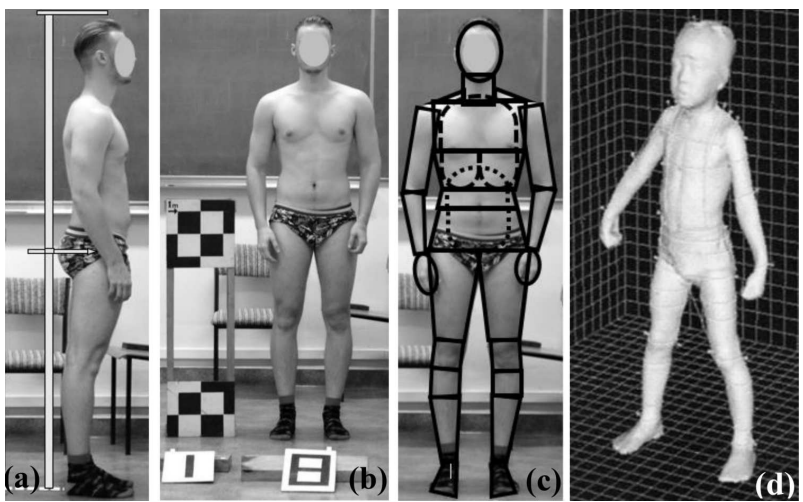

Fig. 1. Different approaches for obtaining external body dimensions using: (a) anthropometer; (b) body image from photograph; (c) regular geometric shapes (dashed line - thorax, i.e. lungs, ribs, dotted line internal tissues of abdomen); (d) image obtained from a laser scanner (courtesy: Hamamatsu).

geometric shapes of body parts with cross-section shape of ellipse (Fig. 1c), (iv) using three-dimensional scanner in order to obtain linear, planar and spatial dimensions of the body and of its segments (Fig. 1d).

\subsection{Internal body geometry}

Up to W.C. Roentgen invention of x-rays (Nobel prize of 1901) internal images of the body were obtained only by cutting cadavers. Next important inventions were computer assisted tomography (CAT) of G.N. Hounsfield and A.M. Cormack (Nobel prize of 1979) and magnetic resonance imaging (MRI) of P. Mansfield and P. Lauterbur (Nobel prize of 2003).

CAT images provide internal geometric dimensions of the trunk (Fig. 2a-c). In order to perform orthopedic operation a surgeon needs detailed dimensions of the human bone morphology. This is in order to prepare bioengineering devices used for replacement, reinforcement, and stabilization (fixation) of bones. They must fit patient bones of different dimensions (Fig. 3a-d).

\section{Inertia of the body \\ 3.1. Inertia of body tissues}

In 1990 Erdmann and Gos published data on 50 trunk tissues [2]. They investigated density of tissues taken from fresh cadavers (up to $24 \mathrm{~h}$ after the death). The age of investigated persons just before the death was between 20 and 40 yrs. The smallest density data was obtained for lung tissue, i.e. $0.563 \mathrm{~g} / \mathrm{cm}^{3}$. Below 1.0 were just few tissues including adipose (fat) tissue, i.e. 0.938 . The majority of tissues had density between 1.0 and 1.2. The highest density was reported by Clauser et al. [3] for compact bone, i.e. $1.892 \mathrm{~g} / \mathrm{cm}^{3}$.

According to Kim et al. [4] male American person (medium age about $52.0 \mathrm{y}$, different origin), had $38 \%$ of muscle tissue and about $33 \%$ of adipose tissue taken total mean body mass of $67.6 \mathrm{~kg}$ as $100 \%$. Female American person (medium age $46.6 \mathrm{y}$, different origin) had $32 \%$



(b)

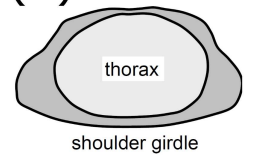

(c)

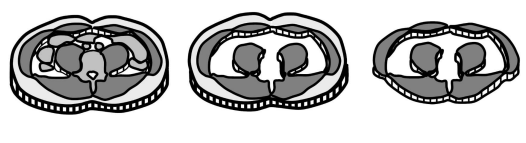

Fig. 2. Examples of images obtained from computerized tomography [1]: (a) a set of layers of $8 \mathrm{~mm}$ width (a ... per) - anthropological landmarks; (b) differentiation of particular body parts on the image of a layer; (c) differentiation of tissues within a layer at the level of umbilicus (from the left: digestion, muscle, and adipose (fat); muscle and adipose; muscle).
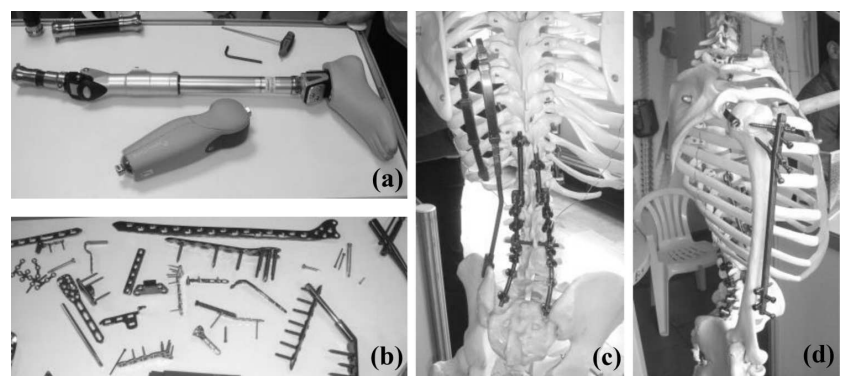

Fig. 3. Biomedical engineering approach to the human body: (a) prostheses fitted to the bone stump; (b) mechanical devices for orthopedic operations; (c) reinforcement of vertebral column and ribs; (d) stabilization of arm bone after breaking.

of muscle tissue and about $54 \%$ of adipose tissue taken total mean body mass of $56.3 \mathrm{~kg}$ as $100 \%$. A trunk has usually about $12 \mathrm{~kg}$ of muscle tissue which constitutes about $35 \%$ of trunk mass and about $4 \mathrm{~kg}$ of adipose tissue which constitutes about $10 \%$ of trunk mass for not obese people [1].

\subsection{Inertia of body parts}

For several years human body is divided on the trunk and the rest of body parts. Since the trunk constitutes about half of body mass it is necessary to divide the trunk on at least few segments. Just few researchers followed this problem. Zatsiorsky and Seluyanov [5] divided trunk onto three parts, Dempster [6] divided onto four parts, Erdmann divided at first onto five and then onto ten parts [7]. Division of the trunk onto several segments is important especially for analysis of movement where the trunk assumes curved configuration, e.g. during gymnastic exercises. 
Mass of body parts differs substantially. Hand has less than 1.0 while abdomen has over $11.0 \mathrm{~kg}$ for a fit, young, adult, male person of mean whole body mass of $80 \mathrm{~kg}$. In relative values hand has below 1.0 and abdomen has about $15 \%$ of the total body mass [8].

Location of center of mass can be obtained using few methods, both direct and indirect. Direct method is based on a lever where measured body is put on a board which is laid on the edge of a triangular prism. Another approach is when a body lies on the reaction board, with one end based on a scale pan. Having total mass of the body and reading of scale data one can calculate location of center of mass from one of the ends (excluding mass of the board) - Fig. 4a and b. Indirect method is based on a photograph of the object. If mass of body parts is known and also locations of centers of mass of every part, it is possible to obtain center of mass of the whole body, e.g. calculating step by step common centers of mass of consecutive adjacent parts.


Fig. 4. Center of mass of the whole body: (a) a body lies on the reaction board in order to calculate position of the center of mass: $D=(W \times L) / Q$; (b) position of location of center of mass $D$ according to the stature $H$; (c) location of the whole body center of mass (wb) according to a hurdle height $(\mathrm{hu})$.

Location of center of mass of parts of extremities is about $43-44 \%$ beginning from the proximal endpoint (closer to the trunk) $[3,6]$. According to $[1,7]$ if the trunk length (between hip joints and base of the neck) is accepted as $100 \%$ then center of mass of the pelvis is $7 \%$, abdomen $40 \%$ and thorax $72 \%$ from the level of the hip joints. Center of mass of the shoulder lies on the line between arm joint and acquired point (65\% from the level of the hip joints) and $41 \%$ from the center of the trunk where half of the trunk's width was accepted as $100 \%$.

Moment of inertia of extremities can be calculated when these parts are acquired as cylinders or a truncated cones. Then radius of inertia is given according to the length of the part. When part length is accepted as $100 \%$ then radii of inertia, e.g. for lower extremities equal: 26.7 for thigh, 27.5 for shank, $24.5 \%$ for foot [9]. According to Erdmann [10] moment of inertia of the whole, straight lower extremity of young, fit, adult males equals (mean \pm S.D.) $3.2 \pm 0.4 \mathrm{~kg} \mathrm{~m}^{2}$.

\subsection{Inertia of the whole body}

Whole body mass of a new born healthy baby equals 3 $4 \mathrm{~kg}$. For adult people extreme mass can be over $500 \mathrm{~kg}$ (for females) and over $600 \mathrm{~kg}$ (for males). On average young adult human beings have body mass of $50-70 \mathrm{~kg}$ (females) and 70-90 kg (males). Density of the whole body is about $1.05 \mathrm{~g} / \mathrm{cm}^{3}$.

Center of mass of the whole erected body lies usually $55-57 \%$ of the body stature (Fig. $4 \mathrm{~b}$ ). It is near the sacrum bone. Every change of body configuration causes change in the position of the center of mass. Center of mass can go outside of the curved body, e.g. during a high jump. Moment of inertia of the whole body changes significantly for example during jumps into the water from a high tower where a jumper changes his or her body configuration thus obtaining different velocity of angular rotations.

\section{Final remarks}

Data on geometric and inertial quantities of the human body are used within several scientific and applied professional areas. These are: everyday living, health, ergonomics, engineering, sport, medicine, forensic, aeronautics, astronautics, and others.

Scientists use full or simplified models of the human body in order to use them in balance and movement analysis, e.g. during lifting of loads, crash tests, sport technique assessment (Fig. 4c), medical use of orthoses and prostheses, and others.

\section{References}

[1] W.S. Erdmann, Research on geometric and inertial quantities of the male trunk obtained by computerized tomography method (in Polish with English abstract), J. Sniadecki University of Physical Education, Gdansk 1995.

[2] W.S. Erdmann, T. Gos, J. Biomech. 23, 945 (1990).

[3] C.E. Clauser, J.T. McConville, J.W. Young, Weight, volume, and center of mass of segments of the human body, Technical Report AMRL-TR-69-70, Aerospace Medical Research Laboratory, Aerospace Medical Division, Air Force Systems Command, WrightPatterson Air Force Base, Ohio 1969. 
[4] J. Kim, Z. Wang, S.B. Heymsfield, R.N. Baumgartner, D. Gallaher, Am. J. Clin. Nutr. 76, 378 (2002).

[5] V.M. Zatsiorsky, V.N. Seluyanov, Voprosy antropologii 62, 91 (1979) (in Russian).

[6] W.T. Dempster, Space requirements of the seated operator: geometric, kinematic, and mechanical aspects of the body with special reference to the limbs, Technical Report WADC-TR-55-159, Wright Air Development Center, Air Research and Development Command, United States Air Force, Wright-Patterson Air Force Base, Ohio 1955.

[7] W.S. Erdmann, J. Biomech. 7, 679 (1997).
[8] R. Kowalczyk, Ph.D. Thesis, Faculty of Physical Education, J. Sniadecki University of Physical Education and Sport, Gdansk 2014.

[9] V.M. Zatsiorsky, A.S. Aruin, V.N. Seluyanov, Biomechanics of the human movement system (in Russian), Fizkultura i Sport, Moscow 1981.

[10] W.S. Erdmann, in: Biomechanics X-B, Proceedings of the Xth International Congress of Biomechanics, Umea, June 15-20, 1985, Ed. B. Jonsson, Human Kinetics Publishers, Champaign (IL) 1987, pp. 10491053. 